\title{
Neutron Decoherence Imaging for Visualizing Bulk Magnetic Domain Structures
}

\author{
C. Grünzweig, ${ }^{1}$ C. David, ${ }^{1}$ O. Bunk, ${ }^{1}$ M. Dierolf,${ }^{1,4}$ G. Frei,${ }^{1}$ G. Kühne, ${ }^{1}$ J. Kohlbrecher, ${ }^{1}$ R. Schäfer, ${ }^{2}$ P. Lejcek, ${ }^{3}$ \\ H. M. R. Rønnow, ${ }^{4}$ and F. Pfeiffer ${ }^{1,4, *}$ \\ ${ }^{1}$ Paul Scherrer Institut, CH-5232 Villigen PSI, Switzerland \\ ${ }^{2}$ IFW Dresden, Institute for Metallic Materials, Helmholtzstrasse 20, D-01069 Dresden, Germany \\ ${ }^{3}$ Institute of Physics, AS CR, CZ-182 21 Praha 8, Czech Republic \\ ${ }^{4}$ Ecole Polytechnique Fédérale de Lausanne, CH-1015-Lausanne, Switzerland
}

(Received 12 April 2008; published 11 July 2008)

\begin{abstract}
Here we introduce a novel neutron imaging method, which is based on the effect that the spatial coherence of the neutron wave front can be changed through small-angle scattering of neutrons at magnetic domain walls in the specimen. We show that the technique can be used to visualize internal bulk magnetic domain structures that are difficult to access by other techniques. The method is transferable to a wide variety of specimens, extendable to three dimensions, and well suited for investigating materials under the influence of external parameters, as, e.g., external magnetic field, temperature, or pressure.
\end{abstract}

DOI: 10.1103/PhysRevLett.101.025504

Magnetic domains and their substructures form the link between the basic physical properties of a magnetic material and its macroscopic characteristics [1]. Today a range of experimental techniques exists for the observation of surface domains (like Kerr microscopy, magnetic force microscopy, or the classical Bitter technique) and for the investigation of domains in thin film samples, provided they are transparent for electrons (Lorentz microscopy) or $\mathrm{x}$ rays (spectro-microscopy) [1,2]. The investigation of internal domains in macroscopic bulk metallic materials, however, still remains a significant challenge.

Since neutrons can easily penetrate centimeter thick metallic samples and interact through their spin directly with the local magnetization [3], several attempts have been made to detect changes in the neutron wave function induced by samples with magnetic domain structures in the past years [4-11]. They can be classified into single-crystal interferometry [4-6], crystal analyzer-based topography $[7,8]$, and neutron depolarization methods $[9,10]$. In practice, however, the existing approaches are limited by several experimental constraints and are therefore presently not available for widespread applications. In the case of neutron crystal interferometry and analyzer-based neutron topography, for example, the most severe practical limitation is imposed by the very small acceptable beam divergence (a few mdeg) and the small energy spread (fractions of $\mathrm{meV}$ ) that are compatible with crystal optics $[5,6]$.

In the following, we present a new approach, based on a grating interferometer, that can overcome these limitations and yield images of bulk magnetic domain structures with polychromatic neutron sources. We have recently shown that such a device can be used for imaging the neutron phase shift induced by the nuclear potential of the sample [12]. Here, we extend our previous approach and experimentally demonstrate how the interaction of the neutron wave field with the magnetic potential of the sample can be used to extract spatially resolved images of the internal magnetic domain structure in ferromagnetic materials.
PACS numbers: 61.05.fg, 03.75.Dg, 77.80.Dj

Figure 1(a) shows our experimental setup. It consists of a source grating $G 0$, a phase grating $G 1$, and an analyzer attenuation grating $G 2$. The source grating $(G 0)$, typically placed close to the neutron beam exit port, is an aperture mask with transmitting slits. It creates an array of periodically repeating line sources and effectively allows the use of relatively large, i.e., square centimeter-sized neutron sources, without compromising the coherence requirements for the arrangement formed by $G 1$ and $G 2[13,14]$, within which the image contrast itself is formed [12]. The grating $G 1$ acts as a phase mask, and imprints periodic phase modulations onto the incoming wave field. Through the Talbot effect, the phase modulation is transformed into an intensity modulation in the plane of $G 2$, forming a linear periodic fringe pattern perpendicular to the optical axis and parallel to the lines of $G 1$, Fig. 1(b). The last grating, $G 2$, with absorbing lines and the same periodicity and orientation as the fringes created by $G 1$, is placed in the detection plane directly in front of the detector. When one of the gratings is scanned along the transverse direction $x_{g}$, the intensity signal $I(m, n)$ in each pixel $(m, n)$ in the detector plane oscillates as a function of $x_{g}$, as shown in Fig. 1(c).

The fundamental idea of the method presented here is to evaluate the local changes of the oscillation $I\left(m, n, x_{g}\right)$ induced by the neutron's interaction with the local magnetic domain structure of the sample. To analyze these changes quantitatively, we write the intensity oscillation for each detector pixel in a Fourier series

$$
\begin{aligned}
I\left(m, n, x_{g}\right) & =\sum_{i} a_{i}(m, n) \cos \left[i k x_{g}+\phi_{i}(m, n)\right] \\
& \approx a_{0}(m, n)+a_{1}(m, n) \cos \left[k x_{g}+\phi_{1}(m, n)\right]
\end{aligned}
$$

where $a_{i}$ are the amplitude coefficients, $\phi_{i}$ the corresponding phase coefficients, $k=2 \pi / p_{2}$, and $p_{2}$ is the period of $G 2$. Then the normalized average transmission of the 
(a)

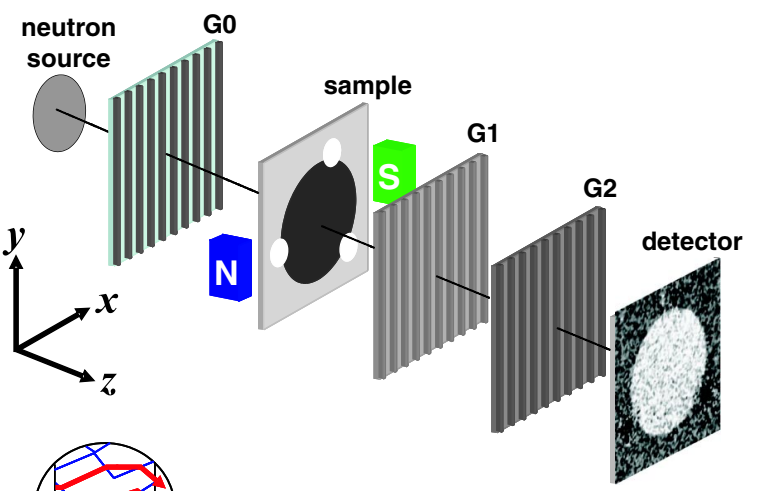

(b)
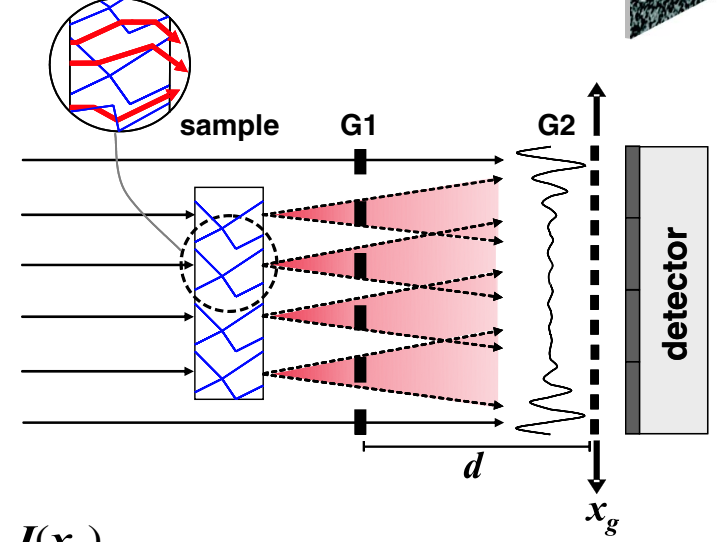

(c)

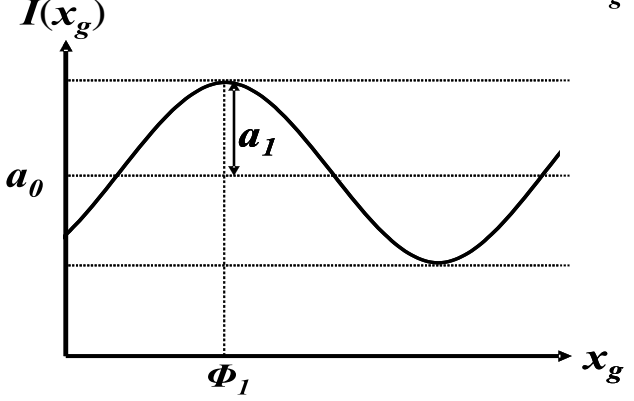

FIG. 1 (color online). Neutron grating interferometer. (a) Setup with a source grating $G 0$, a phase grating $G 1$, and an analyzer absorption grating $G 2$. (b) Through the Talbot effect a linear periodic fringe pattern is created behind $G 1$ in the plane of $G 2$. (c) Intensity modulation detected in a detector pixel when one of the gratings is scanned along $x_{g}$.

specimen in each detector pixel is given by $T(m, n)=$ $a_{0}^{s}(m, n) / a_{0}^{r}(m, n)$, where the superscripts $s$ and $r$ denote the values measured with the specimen in place and as a reference without, respectively. Note that $T(m, n)$ is identical to what would be measured with a conventional neutron radiography setup.

The new aspect of the work presented here is how a detailed analysis of the amplitude of the oscillation, $a_{1}(m, n)$, yields spatially resolved information on the magnetic domain structure in the sample. Our analysis is based on the effect that the neutron beam undergoes multiple refractions at the domain boundaries (domain walls) in the sample [15], resulting in a local degradation of the coherence of the neutron exit wave front behind the specimen [13]. This local degradation decreases the ability of the neutrons to interfere with each other behind the phase grating $G 1$ and yields locally smaller values of the fringe visibility detected in the intensity oscillation $I\left(m, n, x_{g}\right)$ in certain detector pixels [see also Fig. 1(b)].

For a more quantitative description of this effect, we first define the visibility of the intensity modulation of $I\left(m, n, x_{g}\right)$ by the ratio $V^{r}(m, n) \equiv\left(I_{\max }-I_{\min }\right) /\left(I_{\max }+\right.$ $\left.I_{\min }\right)=a_{1}^{r}(m, n) / a_{0}^{r}(m, n)$. The relative decrease of this visibility due to the local coherence degradation caused by the specimen can then be quantified by defining the normalized visibility by $V(m, n) \equiv V^{s}(m, n) / V^{r}(m, n)$. For plane, homogeneous, and nonmagnetic specimens, i.e., for samples that only introduce a constant phase shift due to the neutrons nuclear interaction potential, the value for the visibility remains unchanged, and $V(m, n)=1$. But specimens that exhibit a strongly varying nuclear or magnetic interaction potential show a significant decrease of the visibility with values of $V<1$. More generally, we note that the quantity $V(m, n)$ is an inverse measure of the sample's local ability to degrade the coherence properties of a well-defined neutron wave front through scattering or multiple refraction at density or magnetic potential fluctuations in the sample. Therefore, we will refer to this image in the following as the neutron "decoherence image" (DCI) or "dark-field image" (DFI) of the specimen [16].

The experiments were carried out at the Swiss Spallation Neutron Source (SINQ) using the beam port of the cold neutron imaging facility (ICON [17]). A velocity selector was used to select neutrons with an average wave length of $\lambda \simeq 4.1 \AA$ and a wave length distribution (FWHM) of $\Delta \lambda / \lambda \simeq 16 \%$.
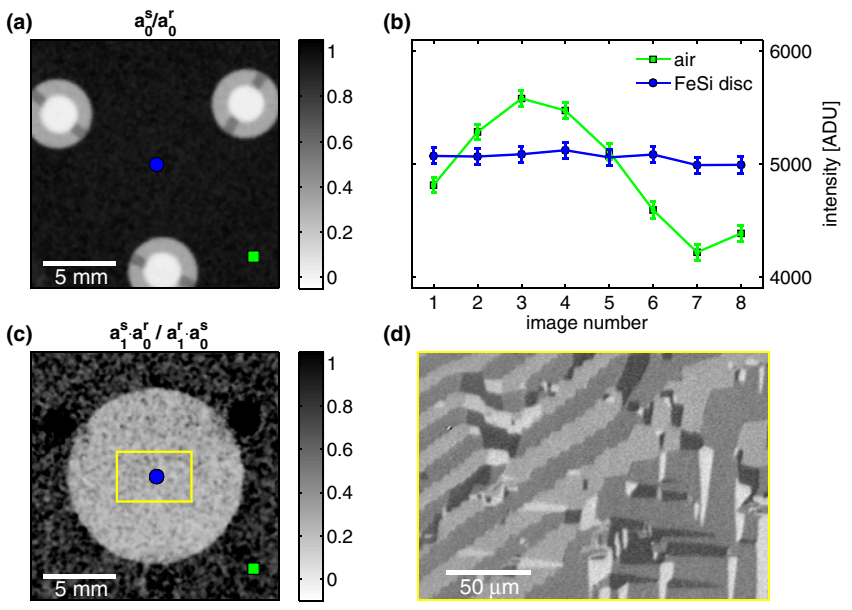

FIG. 2 (color online). Results obtained for a (100)-oriented FeSi test sample. (a) Conventional neutron transmission image (normalized to the empty beam). (b) Intensity oscillations for two detector pixels extracted from a series of eight images taken at different values of $x_{g}$. (c) Neutron decoherence image (DCI) of the same sample (normalized visibility, see text). (d) Corresponding magneto-optical Kerr effect (MOKE) image of a section of the disc, showing the surface domain structure. 
Figure 2 displays results, obtained for a (100)-oriented iron silicon (FeSi) single-crystal disc $[18,19]$. The conventional transmission image is shown in Fig. 2(a) and the corresponding DCI in Fig. 2(c) [21]. While only the strongly attenuating plastic screws used to mount the disc are visible in the transmission image, Fig. 2(a), the FeSi disc is clearly visible in the DCI, Fig. 2(c). This is because the multidomain structure in the sample strongly degraded the coherence of the neutron wave field leading to significantly smaller intensity modulations in $I\left(m, n, x_{g}\right)$, Fig. 2(b), and to correspondingly low values in the DCI [Fig. 2(c)]. The plastic screws, on the other hand, caused no significant contributions to the DCI since the plastic is a homogeneous material with essentially no density or magnetic fluctuations on the relevant length scale. Figure 2(d) shows the surface domain structure of the specimen recorded by magneto-optical Kerr microscopy in the center region of the sample. The zigzag domain walls indicate that the visible domains are closure domains of internal basic domains that are magnetized perpendicularly to the sheet surface ( $V$-line pattern [1]). With a width in the $10 \mu \mathrm{m}$ range, these domains are well below the lateral resolution limit of the imaging detector $(\approx 100 \mu \mathrm{m}[20])$ and thus are not resolved individually in Fig. 2(c) [22].

The efficiency of our setup with total exposure times of typically four minutes per DCI allowed us to study the dynamic response of the specimen under the influence of an externally applied magnetic field, see Fig. 1(a). The corresponding experimental results are shown in Fig. 3. The external magnetic field is applied parallel to the [01 $\overline{1}]$

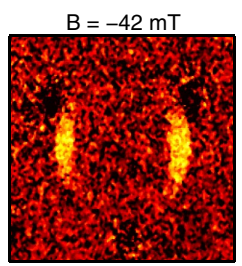

$B=-24 m T$

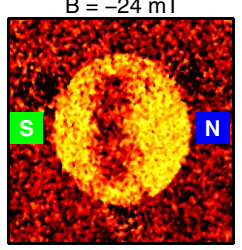

$\mathrm{B}=30 \mathrm{mT}$

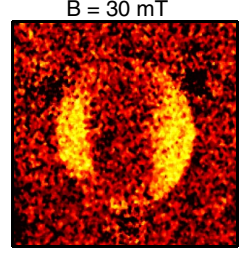

$B=-36 \mathrm{mT}$

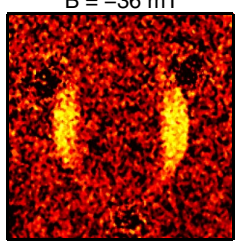

$\mathrm{B}=0 \mathrm{mT}$

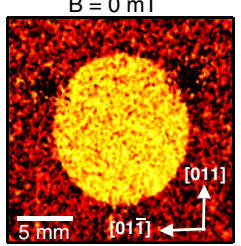

$\mathrm{B}=36 \mathrm{mT}$

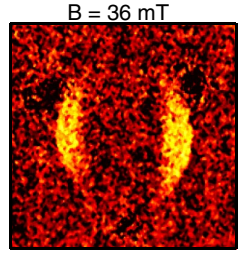

$B=-30 \mathrm{mT}$

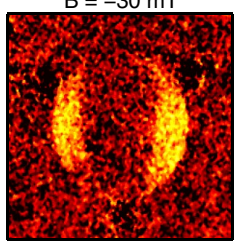

$\mathrm{B}=24 \mathrm{mT}$

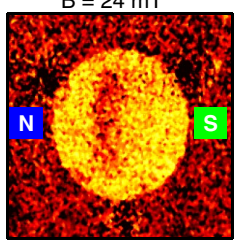

$\mathrm{B}=42 \mathrm{mT}$

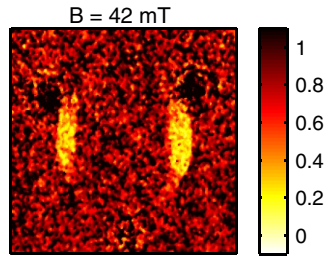

FIG. 3 (color online). Neutron decoherence images of the (100)-oriented FeSi single-crystal disc as a function of a horizontally applied external magnetic field. See also Ref. [24] for an animated series of the magnetization process.

direction of the disc and coincides with a magnetic hard axis of the specimen [23]. For small magnetic field values of $|B| \leq 20 \mathrm{mT}$, the disc is clearly visible in the DCIs, indicating the rich multidomain structure in the specimen that effectively destroys the spatial coherence of the beam. When the magnetic field is increased to values of $|B| \approx$ $24 \mathrm{mT}$, the presence of a vertically oriented zone, which starts growing from the center of the disc, becomes visible in the corresponding DCIs. This zone indicates the presence of a monodomain state of a certain, but unknown, magnetization direction. With increasing field, the zone of saturation expands toward the sample edges that are oriented transverse to the magnetic field axis. Finally, the whole sample becomes fully saturated when $|B| \approx$ $60 \mathrm{mT}$ (see also the animation of the magnetization process in Ref. [24]).

To validate the interpretation of our DCI results, we conducted small-angle neutron scattering (SANS) experiments, which are shown in Fig. 4 [25]. The orientation of the disc and the direction of the external magnetic field was identical to the DCI experiments, described above (Figs. 2 and 3). We indeed observe that the diffraction pattern is broadened due to multiple scattering of neutrons at domain walls in the specimen $[15,26,27]$ when no magnetic field is applied and the sample is in a complex multidomain configuration, Fig. 4(a). When the magnetic field is switched on (to $|B|=60 \mathrm{mT}$ ), the beam broadening (caused by the disturbance of the neutron wave front) disappears and a

(a)
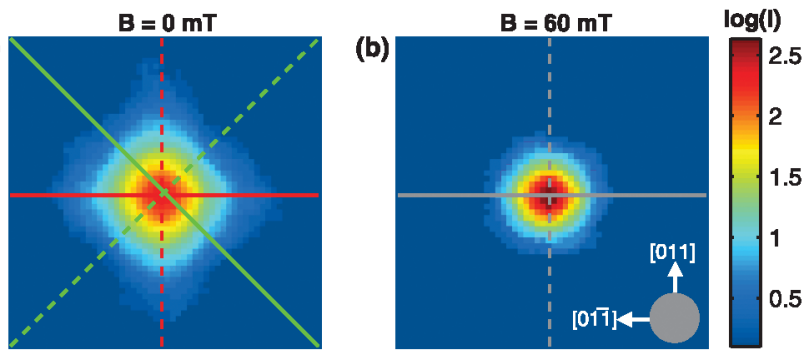

(c)

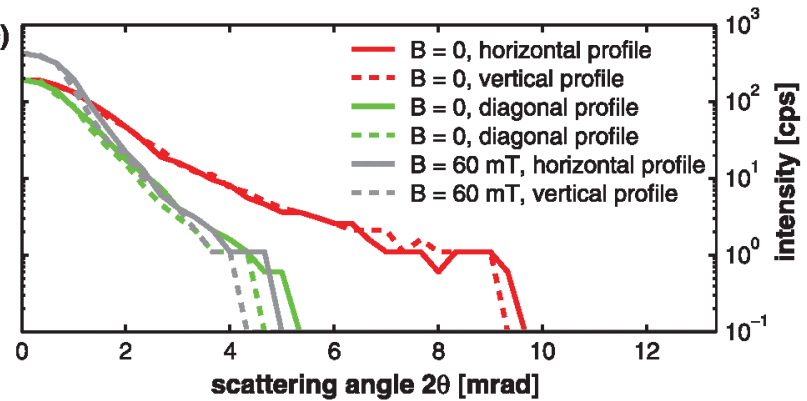

FIG. 4 (color). Small-angle neutron scattering results of the (100)_oriented FeSi single-crystal disc. (a) Scattering pattern without an external magnetic field (logarithmic color scale). (b) Scattering pattern with a horizontally applied external magnetic field of $B=60 \mathrm{mT}$ (logarithmic color scale). (c) Profiles through the scattering patterns without and with the external magnetic field as a function of the scattering angle $2 \theta$. 
beam profile corresponding to the original instrumental resolution is regained, Fig. 4(b).

In conclusion, we have shown how a grating based interferometer can provide spatially resolved images of the magnetic domain structure in centimeter-sized opaque ferromagnetic specimens. Complementary SANS measurements validate our interpretation that the loss of spatial coherence that is revealed in the DCI is caused by smallangle scattering of neutrons at magnetic inhomogeneities in the specimen. Our approach is highly efficient because it is compatible with a broad angular and energy spectrum of the incident neutron beam and does not require inefficient crystal optics. It thus opens the way for systematic investigations of the magnetic properties of bulk materials as a function of, e.g., a variable external magnetic field. We envisage that the method can be extended into 3D using computed tomography and yield three-dimensional images of the magnetic domain wall distributions of bulk ferromagnetic materials. Finally, an implementation of our approach with polarized neutrons potentially allows for three-dimensional structural characterization of the orientation and the magnitude of the local magnetization of an ensemble of magnetic domains in a bulk material [28].

We gratefully acknowledge E. Lehmann for granting us experiment time on ICON, M. Bech and I. Johnson for assistance during the experiments, S. Pofahl for the sample preparation, and L. Heydermann for fruitful discussions.

*franz.pfeiffer@psi.ch

[1] A. Hubert and R. Schäfer, Magnetic Domains (Springer, Berlin, 1998).

[2] H. Hopster and H.P. Oepen, Magnetic Microscopy of Nanostructures (Springer, Berlin, 2005).

[3] O. Halpern and T. Holstein, Phys. Rev. 59, 960 (1941).

[4] M. Schlenker, W. Bauspiess, W. Gräff, U. Bonse, and H. Rauch, J. Magn. Magn. Mater. 15-18, 1507 (1980).

[5] S. Nakatani, H. Tomimitsu, T. Takahashi, and S. Kikuta, Jpn. J. Appl. Phys. 31, L1137 (1992).

[6] H. Rauch and S.A. Werner, Neutron Interferometry (Oxford University Press, Oxford, 2000).

[7] M. Schlenker and J. Baruchel, J. Appl. Phys. 49, 1996 (1978).

[8] J. Baruchel, Physica B (Amsterdam) 192, 79 (1993).

[9] M. T. Rekveldt, Z. Phys. 259, 391 (1973).

[10] S. Mitsuda, H. Yoshizawa, and Y. Endoh, Phys. Rev. B 45, 9788 (1992).

[11] O. Schärpf and H. Strothmann, Phys. Scr. T t24, 58 (1988).

[12] F. Pfeiffer et al., Phys. Rev. Lett. 96, 215505 (2006).

[13] F. Pfeiffer et al., Phys. Rev. Lett. 94, 164801 (2005).

[14] F. Pfeiffer, T. Weitkamp, O. Bunk, and C. David, Nature Phys. 2, 258 (2006).
[15] O. Schärpf, J. Appl. Crystallogr. 11, 626 (1978).

[16] F. Pfeiffer et al., Nat. Mater. 7, 134 (2008).

[17] G. Kühne, G. Frei, E. Lehmann, and P. Vontobel, Nucl. Instrum. Methods Phys. Res., Sect. A 542, 264 (2005).

[18] The (100)-oriented FeSi disc had a thickness of $500 \mu \mathrm{m}$ and was cut from a single-crystal alloy of $\mathrm{Fe}$ (with 2.75 mass \% Si) with a diameter of $12 \mathrm{~mm}$. The bulk single crystal was grown by an rf-heated floating zone technique in hydrogen with a rate of $7 \mathrm{~mm} / \mathrm{h}$.

[19] The gratings were fabricated using standard photo lithography $(G 0, G 1, G 2)$, chemical wet etching $(G 1, G 2)$, and sputtering or evaporation of Gadolinium for $G 0$ and $G 2$ [20]. The gratings were placed with their lines perpendicular to the optical axis of the setup and parallel to the vertical direction. They had periods of $p_{0}=1.08 \mathrm{~mm}$, $p_{1}=7.97 \mu \mathrm{m}$, and $p_{2}=4.00 \mu \mathrm{m}$. The distances between the gratings were $l=5.23 \mathrm{~m}$ and $d=19.4 \mathrm{~mm}$. A circular source (located $1.58 \mathrm{~m}$ from beam port exit) with a diameter of $20 \mathrm{~mm}$ was used.

[20] C. Grünzweig, G. Frei, E. Lehmann, G. Kühne, and C. David, Rev. Sci. Instrum. 78, 053708 (2007); C. Grünzweig, F. Pfeiffer, O. Bunk, T. Donath, G. Kühne, G. Frei, M. Dierolf, and C. David, Rev. Sci. Instrum. 79, 053703 (2008).

[21] To extract the decoherence and transmission signals from the measured intensity modulation $I\left(m, n, x_{g}\right)$, onedimensional discrete fast Fourier transforms (FFT) were computed for each pixel.

[22] The images were recorded using a $100 \mu \mathrm{m}$ thick Li-6/ZnS converter and fluorescence screen with a 1:1 optical lens system and a cooled charge coupled device (CCD) [Fingerlake Instrumentation, $1024 \times 1024$ pixels, pixel size: $24 \times 24 \mu \mathrm{m}^{2}$ ]. The effective spatial resolution was mainly determined by the intrinsic blurring in the scintillation screen to $100 \mu \mathrm{m}$ [20]. A typical exposure time for a single raw image was 30 seconds; typically, four or eight images were taken to process out one decoherence image.

[23] The neutron experiments were performed with a standard dipole electromagnet (GMW associates, C-frame 3470) with plane cylindrical pole pieces of $40 \mathrm{~mm}$ diameter.

[24] See EPAPS Document No. E-PRLTAO-101-049827 for an animation of the experimental data recorded for the magnetization process shown in Fig. 3. For more information on EPAPS, see http://www.aip.org/pubservs/epaps.html.

[25] The SANS experiments (Fig. 4) were carried out at the SANS I instrument of SINQ. A wave length of $\lambda=18 \AA$ (with a $\Delta \lambda / \lambda \simeq 10 \%$ ) was used. The sample detector distance was $20 \mathrm{~m}$, and the size of one detector element was $7.5 \mathrm{~mm}$. The size of the beam at the sample position was $8 \mathrm{~mm}$ in diameter, thus covering most of the central part of the specimen.

[26] A. J. Allen and D. K. Ross, J. Phys. D 17, 99 (1984).

[27] A.Z. Menshikov, S. G. Bogdanov, and Y. N. Skryabin, Physica B (Amsterdam) 234-236, 584 (1997).

[28] H. Leeb, M. Hochhold, G. Badurek, R. J. Buchelt, and A. Schricker, Aust. J. Phys. 51, 401 (1998). 